\title{
Detecção de alterações na camada de fibras nervosas da retina por meio do laser confocal polarizado em hipertensão ocular antes do surgimento de defeitos perimétricos
}

\author{
Detection of retinal nerve fiber layer changes in ocular hypertension with scanning \\ laser polarimetry before the appearance of perimetric defects
}

\author{
Roberto Lauande-Pimentel ${ }^{1,2}$ \\ Keith Barton ${ }^{1}$ \\ Darmaligun Poinoosawmy ${ }^{1}$ \\ Anna Maino ${ }^{1}$ \\ Ted Garway Heath ${ }^{1}$ \\ Roger Hitching's ${ }^{1}$
}

\section{RESUMO}

Objetivo: Avaliar a capacidade do laser confocal polarizado (LCP) em detectar alterações na camada de fibras nervosas (CFN) de hipertensos oculares antes do aparecimento de alteração campimétrica. Desenho- Retrospectivo, caso-controle. Métodos: Pacientes hipertensos oculares divididos em dois grupos: a) estáveis e b) conversores (que progrediram com dano perimétrico glaucomatoso). Parâmetros de retardo obtidos por meio do programa NFA/GDx. Resultados: Um total de 108 pacientes estáveis e 13 conversores foram estudados por período médio de seguimento acima de 35 meses nos dois grupos. Diversos parâmetros do LCP mostraram diferenças significativas na espessura da $\mathrm{CFN}$ entre os dois grupos no inicio do seguimento (média de 27,4 meses antes do aparecimento de lesão perimétrica). Os parâmetros The Number, Maximum Modulation e Superior Average permaneceram diferentes entre os grupos no início e no final do seguimento. O odds ratio para desenvolvimento de conversão perimétrica, dado um resultado de The Number alterado (>32), foi estimado em 7,9 para esta série. Conclusões: O LCP foi capaz de detectar alterações significativas na CFN no grupo de hipertensos oculares que desenvolveram posteriormente lesão perimétrica glaucomatosa. Neste estudo, o resultado inicial anormal de The Number foi o principal fator de risco para desenvolvimento de alteração perimétrica futura em pacientes hipertensos oculares.

Descritores: Glaucoma de ângulo aberto/diagnóstico; Microscopia confocal; Perimetria/ métodos; Fibras nervosas/patologia; Hipertensão ocular/diagnóstico Estudo retrospectivo; Estudos de casos e controle

\section{INTRODUÇ̃̃OO}

2 Pós Graduação, Universidade de São Paulo - USP.

Roberto Lauande Pimentel - obteve suporte para este projeto de pesquisa por meio da Fundação de Amparo a Pesquisa do Estado de São Paulo - FAPESP.

Endereço para correspondência: Roberto Lauande Pimentel - E-mail: rlauande2003@yahoo.com.br Os autores não têm interesse proprietário ou comercial nos produtos citados neste artigo.

Nota Editorial: Pela análise deste trabalho e por sua anuência na divulgação desta nota, agradecemos ao Dr. Michel Eid Farah.

Recebido para publicação em 28.03.2003

Versão revisada recebida em 19.04.2004

Aprovação em 22.04.2004

\footnotetext{
Na prática clínica de glaucoma exames seqüenciais de campo visual automatizado são largamente utilizados para diagnosticar e detectar progressão do dano glaucomatoso ${ }^{(1)}$. Todavia, a perimetria acromática automatizada é relativamente insensível para o diagnóstico precoce do glaucoma ${ }^{(2-4)}$ e além disto é necessário realizar múltiplos exames para se averiguar se uma suspeita de alteração campimétrica é consistente ${ }^{(5-8)}$.

Alterações clínicas e histológicas na camada de fibras nervosas (CFN) da retina foram reportadas cerca de seis anos antes do aparecimento de alterações na perimetria acromática ${ }^{(9-10)}$. $\mathrm{O}$ exame de análise das fibras nervosas da retina por meio do laser confocal polarizado (LCP) é um método
} 
de quantificação indireta, in vivo, da CFN que potencialmente é útil no diagnóstico precoce de glaucoma ${ }^{(11-12)}$. Entretanto, correntemente é necessário obter mais informações para desvendar o potencial desta técnica na detecção de alterações da CFN em pacientes hipertensos oculares (HO) antes que uma lesão perimétrica característica de glaucoma ocorra.

\section{OBJETIVO}

O presente estudo foi desenhado para avaliar a habilidade do LCP na detecção de alterações da CFN em pacientes hipertensos oculares antes da conversão glaucomatosa (surgimento de alteração perimétrica acromática).

\section{MÉTODOS}

Um estudo com desenho retrospectivo longitudinal de pacientes com hipertensão ocular foi realizado, cujos pacientes incluídos foram submetidos a exames periódicos com LCP e CV por um período de pelo menos dois anos.

Após obter aprovação do comitê de ética médica os pacientes hipertensos oculares inscritos na clínica de hipertensão ocular do Moorfields Eye Hospital (Londres, UK), foram identificados.

Os seguintes critérios de inclusão foram adotados: diagnóstico clínico de hipertensão ocular realizado por especialistas do hospital, acuidade visual melhor que 20/30, campos visuais de base normais (vide definição abaixo), exame de LCP com imagens de boa qualidade (veja definição abaixo). Os pacientes com doenças oculares ou/e sistêmicas que pudessem influir nos exames diagnósticos de glaucoma foram excluídos.

O diagnóstico de hipertensão ocular padronizado no hospital inclui os seguintes itens: (a) pressão intra-ocular (PIO) maior que $24 \mathrm{mmHg}$ na tonometria de Goldmann em duas visitas separadas; (b) dois campos visuais normais de base (vide definição abaixo) e (c) discos ópticos de aparência normal em exame biomicroscópico binocular do segmento posterior (lente de 78D).

A análise da perimetria computadorizada acromática padronizada, sendo realizada por intermédio do campímetro Humphrey, estratégia full-threshold, 24-2 (Humphrey Systems, Dublin, USA). Os campos visuais foram considerados confiáveis caso apresentassem: (a) menor que $20 \%$ de perdas de fixação e (b) menor que $33 \%$ falso positivo e falso negativo. Os campos visuais foram considerados normais se: (a) escore AGIS igual a zero ${ }^{(13)} \mathrm{e}$ (b) o Glaucoma Hemifield Test (GHT) normal (Within Normal Limits), em dois exames consecutivos.

A análise longitudinal do campo visual foi realizada por intermédio dos escores AGIS e do programa Progressor ${ }^{\circledR}$ (Progressor for Windows, OBF Labs. Ltd., Malmesbury, UK) que submete os campos consecutivos à análise de regressão linear ponto a ponto conforme detalhado anteriormente ${ }^{(14)}$.

Pacientes estáveis ao longo do estudo foram definidos como os que: (a) persistiram mantendo escores AGIS iguais a zero e (b) cursaram sem demonstrar pontos progressores na análise de regressão linear Progressor ${ }^{\circledR}(\mathrm{p}<1 \%)$.

Os pacientes foram considerados progressores (tendo convertido para glaucoma perimétrico) caso apresentassem: (a) aumento mínimo de quatro pontos na escala AGIS (critério evolutivo utilizado em conformidade com o este estudo) ${ }^{(13)} \mathrm{e} /$ ou (b) dois, ou mais, pontos contíguos significativamente alterados na análise Progressor.

A coleta dos dados do LCP foi realizada por intermédio do aparelho NFAII/GDx (Laser Diagnostic Technologies, San Diego, USA). Três imagens adquiridas independentemente a cada visita foram utilizadas para formar uma imagem média (Mean Image), que serviu de comparação para os exames subsequentes (Baseline Image). Toda a análise foi realizada por um único pesquisador (RLP), que definiu a margem do disco o que automaticamente delineou e congelou a posição de uma elipse de 10 pixels de largura a ser analisada em exames subsequentes, facilitando as comparações longitudinais. Utilizou-se somente imagens que passaram por um controle de qualidade interno do programa GDx (escores de qualidade > 90).

Os parâmetros analisados incluíram: The Number, Symmetry, Superior Ratio, Inferior Ratio, Superior to Nasal, Maximum Modulation, Superior Maximum, Average Thickness, Ellipse Modulation, Ellipse Average, Superior Average, Inferior Average e Superior Integral, todas descritas em detalhes por outros autores ${ }^{(15)}$.

A análise estatística foi feita por meio do software SPSS for Windows (Statistical Package for Social Sciences, versão 9.0, SPSS Inc., Chicago, USA). Variáveis contínuas foram comparadas usando-se o teste $t$ de Student e variáveis categóricas usando o teste qui-quadrado. As alterações longitudinais para cada parâmetro do LCP foram analisadas com o teste $t$ de Student.

\section{RESULTADOS}

Um total de 170 pacientes hipertensos oculares foram considerados inicialmente elegíveis para o estudo. Destes, 49 foram excluídos da análise, pelos seguintes motivos: 5 por anormalidades de campo visual associada a outras patologias ( 2 toxoplasmose, 1 oclusão de veia central da retina, 2 uveíte anterior). Além disto, 20 casos apresentaram dados insuficientes no prontuário e outros 24 casos foram excluídos por não preencherem os critérios de campo visual. Portanto, 121 casos foram analisados, sendo 108 considerados estáveis e 13 apresentaram sinais de progressão (Tabela 1). A idade média foi semelhante entre os dois grupos. O tempo de seguimento foi expressivo e similar nos dois grupos (acima de 35 meses). O número de campos visuais efetuados foi significativamente maior no grupo conversor (Tabela 1).

As características dos grupos no início do estudo mostram que apesar dos campos visuais serem considerados normais por critérios largamente utilizados na clínica (escore AGIS = 0 e GHT normal), uma diferença significativa de MD pode ser 
observada entre os dois grupos. Todavia, em se tratando de pacientes hipertensos oculares, observa-se o baixo valor médio inicial de MD, mesmo no grupo conversor $(-1,00 \pm 2,94)$. Os valores pressóricos, de escavação do disco e CPSD foram semelhantes nos dois grupos (Tabela 2).

$\mathrm{Na}$ análise clínica e perimétrica de progressão longitudinal (Tabela 3), observa-se uma piora significativa nos parâmetros de campo visual (MD, CPSD) e nos parâmetros da análise Progressor (progressing points e slope of progression) no grupo conversor. A pressão ocular e a razão escavação/disco permaneceram similares nos dois grupos. O tempo médio do início do seguimento até a conversão perimétrica foi de 24,7 \pm 5,2 meses.

A tabela 4 demonstra as características do LCP no início do seguimentos para os dois grupos. Observa-se que uma grande parte dos parâmetros indicam diferenças significativas entre os grupos, entre eles destacam-se The Number, Maximum Modulation e Superior Average que permanecem significativamente diferentes entre os grupos do início até o último seguimento, após a conversão perimétrica (Tabela 5).

A subtração dos valores médios dos parâmetros do LCP no final e no início do seguimento estão dispostos na tabela 6 , onde se observa que não ocorreu diferença significativa no ritmo de progressão dos grupos durante o período do estudo.

\section{DISCUSSÃO}

Os resultados deste estudo indicam que desde o início do seguimento os parâmetros do LCP, desenhados para obter medidas globais da CFN (The Number, Maximum Modulation, Average Thickness e Ellipse Average), detectaram diferenças médias significativas entre os grupos de pacientes estáveis e progressores, sugerindo um maior dano inicial a CFN de indivíduos que apresentaram conversão perimétrica posterior. Interessantemente, a exceção de Inferior Ratio, todos os outros parâmetros desenhados para detectar alterações regionais na CFN sugeriram que os setor superior do grupo conversor apresentava diferenças significativa em relação ao grupo estável desde o início do seguimento, conforme os resultados

\begin{tabular}{|lcc|}
\hline \multicolumn{3}{|c|}{ Tabela 1. Dados demográficos dos grupos pacientes Estáveis e } \\
Conversores \\
& $\begin{array}{c}\text { Grupo A } \\
\text { Estáveis } \\
(\mathbf{N}=\mathbf{1 0 8})\end{array}$ & $\begin{array}{c}\text { Grupo B } \\
\text { Conversores } \\
(\mathbf{N}=\mathbf{1 3})\end{array}$ \\
& $62,80 \pm 8,94$ & $66,91 \pm 9,48$ \\
Idade (anos) & $61 / 47$ & $7 / 6$ \\
Sexo (m/f) & $54 / 54$ & $5 / 8$ \\
Olho (direito/esquerdo) & $35,39 \pm 6,93$ & $36,48 \pm 5,65$ \\
Seguimento (meses) & & $11,0 \pm 2,94^{*}$ \\
(média \pm DP) & $10,60 \pm 2,16$ & \\
Número de campos & & \\
visuais & & \\
* $p<0,05$ & & \\
\hline
\end{tabular}

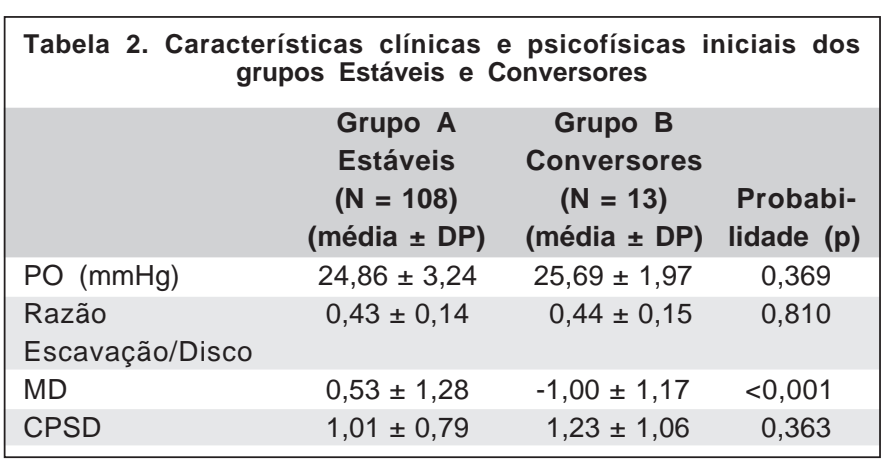

Tabela 3. Alterações clínicas e psico-físicas nos grupos Estáveis e Conversores durante o tempo de seguimento

\begin{tabular}{|c|c|c|c|}
\hline & $\begin{array}{l}\text { Grupo } A \\
\text { Estáveis } \\
(\mathrm{N}=108) \\
\text { (média } \pm \mathrm{DP})\end{array}$ & $\begin{array}{l}\text { Grupo B } \\
\text { Conversores } \\
\quad(N=13) \\
\text { (média } \pm \text { DP) }\end{array}$ & $\begin{array}{l}\text { Probabi- } \\
\text { lidade (p) }\end{array}$ \\
\hline $\begin{array}{l}\text { Diferença em } \\
\text { pressão ocular } \\
(\mathrm{mmHg})\end{array}$ & $-0,83 \pm 4,40$ & $-2,85 \pm 3,58$ & 0,114 \\
\hline $\begin{array}{l}\text { Diferença em } \\
\text { cup/disc ratio }\end{array}$ & $-0,01 \pm 0,09$ & $0,02 \pm 0,10$ & 0,264 \\
\hline Diferença em MD & $0,03 \pm 1,08$ & $-4,00 \pm 2,19$ & $<0,001$ \\
\hline Diferença em CPSD & $0,01 \pm 0,91$ & $2,23 \pm 2,05$ & $<0,001$ \\
\hline $\begin{array}{l}\text { Número de pontos } \\
\text { progressores } \cong\end{array}$ & 0 & $7,92 \pm 6,79$ & $<0,001$ \\
\hline $\begin{array}{l}\text { Índice total de } \\
\text { progressão } \cong\end{array}$ & 0 & $-2,41 \pm 0,90$ & $<0,001$ \\
\hline Escore AGIS & 0 & $4,22 \pm 3,27$ & $<0,001$ \\
\hline \multicolumn{4}{|c|}{$\begin{array}{l}\cong \text { Parâmetro extraído da análise de campo Progressor } \\
\text { As diferenças dos parâmetros foram calculadas por meio da fórmula: } \\
\text { Diferença = (valores do final do seguimento) - (valor do início do seguimento). }\end{array}$} \\
\hline
\end{tabular}

Tabela 4. Valores médios dos parâmetros calculados pelo Laser Confocal Polarizado no início do seguimento

\begin{tabular}{|c|c|c|c|}
\hline Parâmetro & $\begin{array}{c}\text { Grupo A } \\
\text { Estáveis } \\
(\mathrm{N}=108) \\
\text { (média } \pm \mathrm{DP})\end{array}$ & $\begin{array}{c}\text { Grupo B } \\
\text { Conversores } \\
(N=13) \\
\text { (média } \pm \text { DP) }\end{array}$ & $\begin{array}{l}\text { Probabi- } \\
\text { lidade (p) }\end{array}$ \\
\hline The Number & $29,34 \pm 20,19$ & $46,69 \pm 21,24$ & $0,004^{*}$ \\
\hline Symmetry & $1,80 \pm 8,76$ & $0,95 \pm 0,14$ & 0,728 \\
\hline Superior Ratio & $1,97 \pm 0,62$ & $1,59 \pm 0,31$ & $0,032^{*}$ \\
\hline Inferior Ratio & $2,09 \pm 0,43$ & $1,73 \pm 0,55$ & $0,005^{*}$ \\
\hline $\begin{array}{l}\text { Superior to } \\
\text { Nasal Ratio }\end{array}$ & $1,79 \pm 0,26$ & $1,71 \pm 0,36$ & 0,318 \\
\hline Maximum Modulation & $1,35 \pm 0,47$ & $1,03 \pm 0,49$ & $0,023^{*}$ \\
\hline Superior Maximum & $81,01 \pm 14,70$ & $71,08 \pm 9,59$ & $0,019^{*}$ \\
\hline Inferior Maximum & $84,78 \pm 18,17$ & $75,77 \pm 12,01$ & 0,085 \\
\hline Average Thickness & $60,50 \pm 10,54$ & $53,23 \pm 3,76$ & $0,015^{*}$ \\
\hline Ellipse Modulation & $2,11 \pm 0,54$ & $1,97 \pm 0,58$ & 0,383 \\
\hline Ellipse Average & $63,38 \pm 9,77$ & $56,23 \pm 7,73$ & $0,012^{*}$ \\
\hline Superior Average & $69,11 \pm 12,62$ & $58,92 \pm 9,75$ & $0,006^{*}$ \\
\hline Inferior Average & $73,13 \pm 14,09$ & $65,69 \pm 11,79$ & 0,070 \\
\hline Superior Integral & $0,22 \pm 0,22$ & $0,16 \pm 0,02$ & 0,256 \\
\hline
\end{tabular}




\begin{tabular}{|c|c|c|c|}
\hline & $\begin{array}{c}\text { Grupo A } \\
\text { Estáveis } \\
(\mathrm{N}=108) \\
\text { (média } \pm \mathrm{DP})\end{array}$ & $\begin{array}{c}\text { Grupo } B \\
\text { Conversores } \\
(N=13) \\
\text { (média } \pm \text { DP) }\end{array}$ & $\begin{array}{l}\text { Probabi- } \\
\text { lidade } \\
\text { (p) }\end{array}$ \\
\hline The Number & $32,13 \pm 22,67$ & $51,15 \pm 24,33$ & $0,005^{\star}$ \\
\hline Symmetry & $0,97 \pm 0,14$ & $0,95 \pm 0,15$ & 0,630 \\
\hline Superior Ratio & $1,91 \pm 0,55$ & $1,68 \pm 0,48$ & 0,152 \\
\hline Inferior Ratio & $2,01 \pm 0,49$ & $1,81 \pm 0,65$ & 0,183 \\
\hline $\begin{array}{l}\text { Superior to } \\
\text { Nasal Ratio }\end{array}$ & $1,80 \pm 0,34$ & $1,61 \pm 0,34$ & 0,059 \\
\hline Maximum Modulation & $1,27 \pm 0,43$ & $1,03 \pm 0,54$ & 0,067 \\
\hline Superior Maximum & $80,24 \pm 15,47$ & $69,92 \pm 11,93$ & $0,022^{*}$ \\
\hline Inferior Maximum & $83,95 \pm 17,01$ & $74,54 \pm 15,24$ & 0,059 \\
\hline Average Thickness & $60,10 \pm 9,71$ & $55,15 \pm 7,64$ & 0,079 \\
\hline Ellipse Modulation & $1,98 \pm 0,55$ & $1,73 \pm 0,64$ & 0,131 \\
\hline Ellipse Average & $62,24 \pm 11,64$ & $55,77 \pm 8,54$ & 0,055 \\
\hline Superior Average & $68,85 \pm 13,26$ & $57,46 \pm 10,86$ & $0,004^{*}$ \\
\hline Inferior Average & $72,14 \pm 14,27$ & $64,46 \pm 11,85$ & 0,065 \\
\hline Superior Integral & $0,18 \pm 0,03$ & $0,15 \pm 0,03$ & $<0,001^{*}$ \\
\hline
\end{tabular}

\begin{tabular}{|c|c|c|c|}
\hline $\begin{array}{l}\text { Diferença } \\
\text { (valor final - } \\
\text { valor inicial) }\end{array}$ & $\begin{array}{c}\text { Grupo A } \\
\text { Estáveis } \\
(\mathrm{N}=108) \\
\text { (média } \pm \mathrm{DP})\end{array}$ & $\begin{array}{c}\text { Grupo B } \\
\text { Conversores } \\
(N=13) \\
\text { (média } \pm \text { DP) }\end{array}$ & $\begin{array}{l}\text { Probabi- } \\
\text { lidade }(p)\end{array}$ \\
\hline The Number & $2,78 \pm 9,68$ & $4,46 \pm 12,70$ & 0,569 \\
\hline Symmetry & $-0,83 \pm 8,75$ & $0,001 \pm 0,13$ & 0,734 \\
\hline Superior Ratio & $-0,05 \pm 0,46$ & $0,09 \pm 0,28$ & 0,286 \\
\hline Inferior Ratio & $-0,07 \pm 0,43$ & $0,09 \pm 0,46$ & 0,211 \\
\hline $\begin{array}{l}\text { Superior to } \\
\text { Nasal Ratio }\end{array}$ & $0,006 \pm 0,26$ & $-0,10 \pm 0,15$ & 0,153 \\
\hline Maximum Modulation & $-0,07 \pm 0,36$ & $0,001 \pm 0,37$ & 0,504 \\
\hline Superior Maximum & $-0,77 \pm 9,63$ & $-1,15 \pm 7,61$ & 0,891 \\
\hline Inferior Maximum & $-0,83 \pm 13,42$ & $-1,23 \pm 11,45$ & 0,918 \\
\hline Average Thickness & $-0,34 \pm 8,74$ & $1,92 \pm 9,96$ & 0,387 \\
\hline Ellipse Modulation & $-0,13 \pm 0,51$ & $-0,24 \pm 0,73$ & 0,486 \\
\hline Ellipse Average & $-1,14 \pm 8,82$ & $-0,46 \pm 5,45$ & 0,787 \\
\hline Superior Average & $-0,25 \pm 8,92$ & $-1,46 \pm 5,30$ & 0,634 \\
\hline Inferior Average & $-0,99 \pm 8,53$ & $-1,23 \pm 7,93$ & 0,923 \\
\hline Superior Integral & $-0,03 \pm 0,22$ & $-0,001 \pm 0,01$ & 0,637 \\
\hline
\end{tabular}

de Superior Average, Superior Maximum e Superior Ratio (Tabela 4).

Vale a pena ressaltar que o valor médio MD também foi diferente desde o início do seguimento entre os dois grupos $(p<0,001)$. A tabela 3 mostra que com o seguimento a diferença de MD se intensificou, chegando a uma média de $-4,00 \pm 2,19$ no grupo conversor, em consonância com o surgimento de defeito perimétrico neste grupo. Porém, o valor médio inicial de MD no grupo progressor foi de apenas $-1,00 \pm 1,17$ (Tabela
2), sendo obviamente insuficiente para classificar clinicamente um indivíduo como tendo defeito perimétrico significativo.

Utilizando-se o parâmetro The Number, com o ponto de corte para anormalidade $>32$, conforme sugerido anteriormente ${ }^{(16)}$, observa-se que este parâmetro classificou como anormal $31,5 \%(n=34)$ dos pacientes no grupo estável e 69,2\% ( $n=9)$, no grupo conversor no início do seguimento. A mesma estratégia, quando utilizada no final do seguimento, detectou alterações em 37,5\% (n=41) dos pacientes estáveis e 76,9\% (n=10) dos pacientes progressores. Desta forma, a análise simplificada para segregar hipertensos oculares conversores e estáveis apenas com The Number incluiria 31,5\% "falsos positivos" e $30,8 \%$ "falsos negativos"- o que parece ser insuficiente. Todavia, baseado nestes mesmos dados o "odds ratio" estimado para conversão, dado um resultado anormal de The Number é de 7,92 (2,04-30,68), o que sugere que The Number alterado é fator considerável de risco para conversão perimétrica futura.

Este resultado nos parece relevante para na prática clínica, uma vez que apesar da taxa de conversão anual para glaucoma ser estimada em cerca de $1 \%{ }^{(17)}$ os pacientes hipertensos oculares são comumente submetidos a avaliações periódicas, com custo relevante. As diretrizes que determinam a frequência dos exames e o início ou não do tratamento clínico levam muitas vezes em conta os diversos fatores de risco associados, dentre eles, a história familiar, doenças sistêmicas e espessura corneal. Apesar de uma casuística pequena de pacientes conversores, este estudo sugere que o resultado anormal de The Number também pode ser levado em consideração como fator de risco para conversão futura.

A análise de diferença de espessura final e inicial da CFN (Tabela 6), demonstrou que o ritmo de progressão dos dois grupos foi similar e que as diferenças de espessura média da CFN dos dois grupos já eram expressivas desde o início do seguimento. Estes dados são compatíveis com relatos ${ }^{(2)}$ que estimaram um dano de até $50 \%$ nas fibras da CFN, para que o campo visual apresentasse alterações, além de relatos anteriores que sugerem que alterações da CFN podem ser encontradas vários anos antes de alterações perimétricas ${ }^{(9-10)}$. Os resultados da presente série são compatíveis com estes relatos prévios, visto que se observou que LCP foi capaz de detectar alterações significativas da CFN de hipertensos oculares, em torno de vinte e cinco meses antes da detecção de dano glaucomatoso perimétrico.

Estudos anteriores realizados em amostras de hipertensos oculares sugerem que a polarimetria é capaz de detectar alterações significativas em hipertensos oculares quando comparados a controles normais, apesar de considerável sobreposição de valores entre os grupos ${ }^{(18-19)}$. O acompanhamento seqüencial de exames polarimétricos em uma amostra de indivíduos normais e hipertensos oculares indicou um afilamento setorial e global na espessura média de hipertensos oculares com o tempo ${ }^{(20)}$. Calculou-se que, durante o estudo, The Number variou $0,07 / \mathrm{mês}$ no grupo normal e $0,017 /$ mês em hipertensos oculares $(\mathrm{p}<0,001)$.

Uma possível limitação do presente estudo foi a definição 
arbitrária de progressão campimétrica adotada, apesar deste tema ser objeto de grande discussão e pouco consenso na literatura. De fato, os escores utilizados para se estimar progressão nos grandes estudos multicêntricos variam, e não são concordantes em uma série de $\operatorname{casos}^{(21)}$. Especula-se que na prática clínica o escores como o utilizado pelo estudo AGIS possam subestimar alguns casos de progressão localizada ${ }^{(21-22)}$, fato que nos levou a utilizar também o programa Progressor ${ }^{\oplus}$ que efetua análise ponto a ponto, o que teoricamente pode ter aumentado a capacidade para o diagnóstico de progressão.

Além disto, o fato de não haver consenso sobre a melhor forma de definir progressão perimétrica torna importante e útil o estudo de outros métodos independentes (quantitativos e reprodutíveis), tal qual o LCP, que possam auxiliar determinação de progressão glaucomatosa genuína.

Em conclusão, neste estudo observou-se que diversos parâmetros do LCP são capazes de detectar alterações na CFN de hipertensos oculares que evoluíram com glaucoma perimétrico, em torno de 25 meses antes da conversão perimétrica. $\mathrm{O}$ estudo sugere que hipertensos oculares com resultados anormais de The Number apresentam maior risco de conversão futura para glaucoma, todavia novos estudos prospectivos com maior número de pacientes conversores devem ser realizados para ratificar este achado.

\section{ABSTRACT}

Objective: To evaluate the ability of the Confocal Scanning Laser Polarimeter (SLP) to detect glaucoma alterations before the appearance of perimetric defects. Design- retrospective, case-control. Methods: Ocular hipertensive patients divided in to two groups: a) stable and b) conversors (that have conversed to perimetric defined glaucoma). Nerve Fiber Analyser/GDx parameters of retardation. Results: A total of 108 stable and 13 conversors were evaluated for a mean period over 35 months in each group. At the initial examination, several SLP parameters detected significant differences retinal nerve fibre layer thickness in stable and converter groups (a mean of 27.4 months before the appearance of perimetric lesion). The Number, Maximum Modulation and Superior Average remained different between the groups at the initial and final examination. The odds ratio for perimetric conversion, giving a altered The Number result (>32), was estimated at 7.9 in this series. Conclusion: The SLP was capable of detecting significant RNFL alterations in the group of ocular hipertensive patients who developed a characteristic glaucomatous perimetric lesion. In this study an initial abnormal The Number result was a significant risk factor for the development future glaucomatous perimetric defect in ocular hipertensive patients.
Keywords: Glaucoma, Open-Angle/diagnosis; Microscopy, Confocal; Perimetry/*methods; Nerve fibers/pathology; Ocular hypertension/diagnosis; Retrospective study; Case-Control Studies

\section{REFERÊNCIAS}

1. Tuulonen A, Lehtola J, Airaksinen PJ. Nerve fiber layer defects with normal visual fields. Do normal optic disc and normal visual field indicate absence of glaucomatous abnormality? Ophthalmology. 1993;100(5):587-8.

2. Quigley HA, Addicks EM, Green WR. Optic nerve damage in human glaucoma. III. Quantitative correlation of nerve fiber loss and visual field defect in glaucoma, ischemic neuropathy, papilledema, and toxic neuropathy. Arch Opththalmol. 1982;100(1):135-46.

3. Quigley HA, Katz J, Derick RJ, Gilbert D, Sommer A. An evaluation of optic disc and nerve fiber layer examinations in monitoring progression of early glaucoma damage. Ophthalmology. 1992;99(1):19-28.

4. Zeyen TG, Caprioli J. Progression of disc and field damage in early glaucoma. Arch Ophthalmol. 1993;111(1):62-5.

5. Katz J, Sommer A. A longitudinal study of the age-adjusted variability of automated visual fields. Arch Opthalmol. 1987;105(8):1083-6.

6. Werner EB, Petrig B, Krupin T, Bishop KI. Variability of automated visual fields in clinically stable glaucoma patients. Invest Opthalmol Vis Sci. 1989; 30(6):1083-9

7. Zulauf M, Caprioli J. What constitutes progression of glaucomatous visual field defects? Semin Ophthalmol. 1992;7(2):130-46.

8. Fitzke FW, Hitchings RA, Poinoosawmy D, McNaught AI, Crabb DP. Analysis of visual field progression in glaucoma. Br J Opthalmol. 1996;80 (1):40-8.

9. Hoyt WF, Newman NM. The earliest observable defect in glaucoma ? Lancet. 1972;1(7752):692-3.

10. Sommer A, Katz J, Quigley HA, Miller NR, Robin AL, Richter RC, Witt KA. Clinically detectable nerve fiber atrophy precedes the onset of glaucomatous field loss. Arch Ophthalmol. 1991;109(1):77-83.

11. Tjon Fo Sang MJ, Lemij HG. The sensitivity of nerve fiber layer measurements in glaucoma as determined with scanning laser polarimetry. Am J Ophthalmol. 1997;123(1):62-9.

12. Weinreb RN, Zangwill L, Berry CC, Bathija R, Sample P. Detection of glaucoma with scanning laser polarimetry. Arch Ophthalmol. 1998;116(12):1583-9.

13. Advanced Glaucoma Intervention Study. 2. Visual field test scoring and reliability. Ophthalmology. 1994;101(8):1445-55.

14. Fitzke FW, Hitchings RA, Poinoosawmy D, MacNaught AI, Crabb DP. Analysis of visual field progression in glaucoma. Br J Ophthalmol. 1996;80 (1):40-8.

15. Weinreb RN, Zangwill L, Berry CC, Bathija R, Sample PA. Detection of glaucoma with scanning laser polarimetry. Arch Ophthalmol. 1998;116(12): 1583-9.

16. Lauande-Pimentel R, Carvalho RA, Oliveira HC, Gonçalves DC, Silva LM, Costa VP. Discrimination between normal and glaucomatous eyes with visual field and scanning laser polarimetry measurements. Br J Ophthalmol. 2001;85 (5):586-91

17. David R, Livingston DG, Luntz MH. Ocular hypertension- a long-term followup of treated and untreated patients. Br J Ophthalmol. 1979;61(11):668- 74.

18. Anton A, Zangwill L, Emdadi A, Weinreb RN. Nerve fiber layer measurements with scanning laser polarimetry in ocular hypertension. Arch Opththalmol. 1997;115(3):331-4.

19. Tjonf-Fo-Sang MJ, de Vries J, Lemij HG. Measurement by nerve fiber analyser of retinal nerve fiber layer thickness in normal subjects and patients with ocular hypertension. Am J Opththalmol. 1996; 122(2):220-7.

20. Poinoosawmy D. Changes in the thickness of nerve fibre layer with time. Presented in part at: Association for Research in Vision and Ophthalmology. ARVO Annual Meeting; 2000.

21. Katz J, Congdon N, Friedman DS. Methodological variations in estimating apparent progressive visual field loss in clinical trials of glaucoma treatment. Arch Ophthalmol. 1999;117(9):1137-42.

22. Katz J. Scoring systems for measuring progression of visual field loss in clinical trials of glaucoma treatment. Ophthalmology. 1999;106(2):391-5. 